\title{
Promoting Time-Efficient Care for Suicidal Patients in the Emergency Department
}

\author{
Lena Kazanchyan ${ }^{1,2}$, Raymund Gantioque ${ }^{1,2}$, Donovan Stewart ${ }^{1,2}$ \\ ${ }^{1}$ Department of Nursing, California State University of Los Angeles, Los Angeles, CA, USA \\ ${ }^{2}$ Emergency Department, Adventist Health Glendale, Glendale, CA, USA \\ Email:1kazanc@calstatela.edu,rgantio@calstatela.edu,stewardk@ah.org
}

How to cite this paper: Kazanchyan, L., Gantioque, R. and Stewart, D. (2020) Promoting Time-Efficient Care for Suicidal Patients in the Emergency Department. Open Journal of Emergency Medicine, 8, 31-38. https://doi.org/10.4236/ojem.2020.81004

Received: February 13, 2020

Accepted: March 3, 2020

Published: March 6, 2020

Copyright $\odot 2020$ by author(s) and Scientific Research Publishing Inc. This work is licensed under the Creative Commons Attribution International License (CC BY 4.0).

http://creativecommons.org/licenses/by/4.0/ (c) (i) Open Access

\begin{abstract}
Patients who present to an emergency department are in a vulnerable state of mind. The population in the emergency department is continuously growing. Acute stages of mental health problems can bring patients to the emergency department for care. Psychiatric disorders such as schizophrenia, living alone, depression, hopelessness, previous suicide attempts, self-devaluation, agitation, and insomnia are some risk factors that predispose individuals to suicide. Suicidal patients' care in the emergency department can be challenging for healthcare professionals due to the complex environment and numerous other emergencies occurring altogether. Suicidal patients do not get the timely medical attention they need from clinicians. As a result, there are little information and few protocols in emergency departments about the correlation of the timely care and how it can affect the patients during the emergency department visit.
\end{abstract}

\section{Keywords}

Suicide, Suicidal Ideation, Emergency Department (ED), Throughput, Boarding, Nurse Practitioners

\section{Introduction}

Suicide has been the tenth leading cause of death in the United States since 2008 (Centers for Disease Control and Prevention) [1]. Mental health chief complaints constitute for $12.5 \%$ of ED visits [2]. Studies found that more than 500,000 people present to an emergency department (ED) each year with self-harm or suicidal ideations [3]. The emergency department is a primary gateway to many patients, accounting for more than 500,000 people with suicidal ideation. Suicide is a global phenomenon that accounts for 45,000 deaths occurring in the United 
States in 2016 [3]. Suicide attempts and ideations justify a significant amount of emergency department visits. It can offer substantial suicide prevention services by coordinating care with transfers, medications, treatments, and inpatient stays. Early recognition and screening for this population is beneficial during the triage process. However, suicidal patients end up boarding for several hours in emergency departments awaiting evaluation or psychiatric placement without appropriate treatment [4]. Studies indicate that screening patients will help with immediate actions and safety planning, ongoing assessments, evidence-based treatment, and the significance of patient compliance [5]. Nevertheless, care can be delayed from throughput challenges. Therefore, preventing delayed care and prioritizing psychiatric patient care during the acute phase will result in better outcomes and decrease the risk of self-harm.

The objective of this paper is to produce standardized procedures in emergency departments to expedite medical care for suicidal patients that present to the emergency room for help. By emphasizing the importance of time-efficient care, suicidal patients will experience reduced boarding times, thus resulting in a treatment productivity.

\section{Case Study}

A 20-year-old female was brought to a Southern California emergency department by paramedics after being found unconscious by her mother in her house with a pill bottle in sight. She presented with shallow respiration; 11 breaths per minute, blood pressure 111/72 and heart rate 126 beats per minute. She moves all extremities and opens her eyes when reacting to the sternal rub, however does not follow commands. She does not have any focal deficits when assessing a neurological exam. The bottle is a prescription for sertraline, which was filled by her mother three days ago. The friend who called 911 states that the patient is unemployed at this time, had a recent break-up, and has been living at her mother's house for the past week. She was intubated on the scene by paramedics for decreased level of consciousness and transported to the local emergency department. During her stay in the emergency department, she was assessed immediately by the physician. He ordered propofol sedation and started her on a norepinephrine drip to support her blood pressure. After stabilizing her vital signs, her psychiatrist came to visit her 14 hours later. He did not put any orders for this patient. She was admitted to ICU 26 hours later. Her records show no medical history or emergency visits. Three days later, she is extubated and awake. On the fourth day, she is transferred to the psychiatric medical surgical unit where she is being monitored and beginning psychiatric treatment. After interviewing her, it was found that the psychiatrist diagnosed her with major depression and anxiety. Psychiatric medication treatment was started 5 days after her initial admission to the emergency department. Upon discharge, she will be assigned to a mental health clinic for social services and follow up care with counseling. (An informed consent was obtained from the patient to present this 
case).

\section{Suicide Rates}

In the United States, nearly thirty thousand people die annually from suicide [6]. The rate of suicides in California has been growing more than $50 \%$ in the last two decades. The suicide surveillance in California is working on improvement, however, it does take two to three years to collect accurate data due to the verification and preparing process. There were 2358 suicides in the Los Angeles county between 2008 and 2010. Suicide rates in California are higher among the Caucasians as compared with African Americans [7]. In the age group of 10 - 34, suicide is the second leading cause of death and in the ages 35 - 54, it is the fourth leading cause of death [8].

\section{Morbidity and Mortality}

Suicide rates are continuing to rise. Mental health is a growing global phenomenon that is resulting in increased rates of morbidity and mortality. According to the Centers for Disease Control and Prevention, in 2016, nearly 45,000 suicides occurred in the United States among people above the age of 10 [9]. The rates of emergency department visit for nonfatal self-harm have increased; from 2001 to 2016, there has been a $42 \%$ increase [9]. It is known that suicidal ideation and attempted suicide are more dominant in adults with more than one mental disorder and the presence of comorbid psychiatric disorders is related to the increased risk of suicidal ideation [10]. Whether or not a suicide attempt fails or succeeds, this factor has a correlation between the psychotic disorder and the suicidal thought [10].

\section{Identification of Suicidal Patients}

The Joint Commission, a crediting agency for healthcare facilities, has a goal to assist all healthcare organizations to better identify these patients. The Joint Commission requires a suicide screening and an environmental risk assessment for these patients [4]. Clinicians in the emergency department have a crucial role of identifying the patients at risk. The Behavioral Health Care National Safety Goal number 15 is an organization that identifies the safety risks inherent in the population that it serves [11]. In 2018, the Joint Commission revised their Behavioral Health Care National Safety goal, focusing on action [11]. Ordering proper treatment in a timely manner is what these patients need. Many times they come to the emergency department for medication stabilization. Nevertheless, emergency departments are considered primary health care centers for many people. It is easily accessible and the quickest way to seek medical attention. Caring for patients with suicidal thoughts and psychotic behaviors can be difficult especially when unknown outcomes of interventions are provided. However, many organizations do not have adequate protocols and resources, leading to under detection and prolongation of treatment [12]. 


\section{The Scope of the Problem}

Due to the lack of access to resources, suicidal patients find that going to the ED is the fastest way to seek help. Many factors contribute to the scope of the problem. The first being the prolonged stay in the emergency department. This often relates to lack of access to healthcare. This lack of access or admittance to mental health clinics and substance abuse treatment centers is considered a significant risk factor for the suicide behavior development. For example, individuals who lack access or treatment for their substance use behavior, will only be affected further and may consider suicide since their psychological well-being is greatly affected through observation of no meaning in life. Because this population is using the ED as a primary resource center, the goals of the ED setting should be prioritization. The immediate focus should be maintaining safety in these patients and promoting well-timed care.

Another significant problem is boarding patients. "ED boarding" is when patients are to remain in the ED exceeding 24 hours [13]. Prolonged boarding in the ED for psychiatric patients is associated with low-quality care and contributes to crowding [14]. Understanding that boarding can lead to increasing costs, it is an estimated cost of $\$ 2264$ to stay in the ED while waiting for admission [13]. While these patients are boarded, the biggest problem is the barrier to their care. They are not receiving appropriate treatment when waiting in the emergency department. The Emergency Medicine International studied data of how boarding psychiatric patients resulted in an increase in poor outcomes, such as compromising the safety of other patients that are in the emergency department [15]. The American College of Emergency Physicians concluded a survey that reported patients can wait up to two days to be transferred to an inpatient bed, which is a concerning occurrence [16]. Boarding patients in the emergency department prolong the time in which all patients wait for a service. Psychiatric treatment consists of productive group therapy, individual therapy, medications, activities, and counseling, which does not exist in ED during the boarding process.

Moreover, the immense amount of resources that are being used for these patients results in poor clinical outcomes. These outcomes have evidence in delaying care and increasing morbidity and mortality rates, which contribute to the fact of ED overflow and lack of availability to other patients' care [12].

Most inpatient psychiatrists do not provide consults in the emergency department, which means there is no treatment initiation [17]. Inpatient orders are the medical treatment. If the patient is waiting in the ED without adequate medical treatment, then the patient remains "unstable", and behavioral symptoms progress leading to self-harm and suicidal ideations. Thus, this contributes to the growing problems in the ED.

\section{Discussion and Implementation}

How must we resolve these challenges? Mental healthcare demand is increasing. 
More people are utilizing the ED as a primary healthcare center to seek medical attention. Suicide contemplations are far too common in the emergency department [18]. The most important key factor is coordinating care within providers and departments. According to the National Institute of Mental Health, many ED physicians and providers are particularly concerned about excess boarding time for a patient waiting for an inpatient psychiatry bed or transfers [19]. For providers to understand the severity of these high-risk patients, these patients should be screened during triage.

A way to begin implementation is understanding how to recognize these patients. Once patients are identified as suicidal in triage and interviewing, the "intake" process should begin. The intake process should take between $8-12$ minutes while an alert is being communicated through the charge nurse, security, and emergency medical technicians. The process will consist of the patient being taken into an "intake room" where their belongings will be assessed. Simultaneously, a psychiatric nurse will be interviewing the patient and completing the screening tool. Evidence shows that mental health professionals who are trained to work with suicidal patients have a vital role in preventing suicide death [7]. All staff members who are supposed to be Crisis Prevention Institute (CPI) are certified to be able to manage disruptive behavior. Nevertheless, there should be a psychiatric nurse every shift who can determine if the patient needs to be placed on a hold. This will expedite the transfer and admission process. If psychiatric registered nurses are able to put patients on holds, this will promote time efficiency and prevent delayed care. The intake room should be free of dangerous objects, including monitor cords, IV poles, curtains, and other potentially harmful items that can be of risk to their safety. The patient should remain in the intake room with staff members until the ED bed is ready.

Patients who are in the intake process are given green colored hospital gowns to easily be identified by the hospital staff. Once taken into a room, the door would have a logo, particularly a green triangle, identifying them as a high risk patient. The patient is required to have one to one monitoring with a trained emergency support staff at all times. Patients will first be medically clear and then behavioral health treatment and placement should take place. A complete lab workup should be started as soon as the patient gets to the ED room. The ED room should be free of any type of objects such as monitor cords, IV kit's, needles, or anything that the patient can use to cause self-harm. Reflecting on the patient in the case study, hypothetically speaking, if the patient was awake and was actively having suicidal thoughts and had not been intubated, medication administration and physical safety should be two of the top priorities in these patients.

In order to detect and manage suicidal patients, early identification and communication through the healthcare team members is of importance. Having a provider, such as a nurse practitioner, should directly admit the patient under their care to begin treatment to promote throughput and time-efficient care. A 
nurse practitioner is proven to improve patient satisfaction, increase efficiency and timeliness of the ED throughput [20]. With the development of effective measures for treating mental disorders, nurse practitioners will be able to make follow-ups on their patients in the emergency department [21]. Medications should be administered to these patients in the acute phases, which is a crucial component in the treatment process. Providers in the emergency department need to be able to initiate medications to stabilize these patients so that they have treatment before being transferred to the floor. This will promote the throughput process and will demonstrate safe, time-efficient care. As discussed, the development of the workforce will be needed to promote these interventions.

The American College of Emergency Physicians mention a telepsychiatry service that should be available in emergency departments [22]. This is a service that provides video conferencing, which has been implemented in hospitals in the state of Georgia, and has shown a more timelier approach when having access to a psychiatrist. This will prevent patients with mental health issues from waiting for time-efficient care. In a National Alliance on Mental Illness survey, $70 \%$ of patients waited more than 10 hours to see a psychiatrist in the ED [22]. Advantages to having telepsychiatry would be low cost, easy implementation, less stigma when mixed with all patients, and most importantly, a more time-efficient approach to developing the plan of care [16]. Telepsychiatry will help hospitals reduce boarding times, improve throughput, reduce patient waiting time, and lower the risk of elopement and injury [15]. This kind of psychiatric care can improve boarding by determining which patients are critical to be admitted and those who are safe to be discharged with appropriate services. A health system in North Carolina implemented telepsychiatry and saw a reduction in length of stay from 48 hours to 22.5 hours during the entire hospitalization [15].

\section{Conclusion}

Suicide is among the leading causes of death and disability in the human population across the world. Suicide rate is increasing, but emergency medicine is growing and will continue to grow by expediting the quality of patient care. Through a systematic and evidence-based approach, emergency providers can prevent injury and promote fast, efficient care. Focusing on isolating the appropriate patients and helping other staff members easily identify these patients can result in greatly improved outcomes. Implementing telepsychiatry will improve throughput and provide safe, patient-centered care. These findings will be useful for many emergency departments to guide appropriate intervention and improve healthcare quality.

\section{Conflicts of Interest}

The authors declare no conflicts of interest regarding the publication of this paper. 


\section{References}

[1] Hedegaard, H., Curtin, S.C. and Warner, M. (2018) Suicide Mortality in the United States, 1999-2017. NCHS Data Brief No. 330, September 2018. https://www.cdc.gov/nchs/products/databriefs/db330.htm

[2] Chang, B., Tezanos, K., Gratch, I. and Cha, C. (2019) Depressed and Suicidal Patients in the Emergency Department: An Evidence-Based Approach (Behavioral Health CME). EB Medicine.

https://www.ebmedicine.net/topics/psychiatric-behavioral/depression-suicidal\#Intr oduction

[3] National Institute of Mental Health (13 December 2019) Emergency Department Study Reveals Patterns of Patients at Increased Risk for Suicide.

https://www.nimh.nih.gov/news/science-news/2019/emergency-department-study-r eveals-patterns-of-patients-at-increased-risk-for-suicide.shtml

[4] Betz, M.E. and Boudreaux, E.D. (2016) Managing Suicidal Patients in the Emergency Department. Annals of Emergency Medicine, 67, 276-282.

https://www.ncbi.nlm.nih.gov/pmc/articles/PMC4724471/ https://doi.org/10.1016/j.annemergmed.2015.09.001

[5] King, C.A., Horwitz, A., Czyz, E. and Lindsay, R. (2017) Suicide Risk Screening in Healthcare Settings: Identifying Males and Females at Risk. Journal of Clinical Psychology in Medical Settings, 24, 8-20. https://doi.org/10.1007/s10880-017-9486-y

[6] Bolster, C., Holliday, C., Oneal, G. and Shaw, M. (2015) Suicide Assessment and Nurses: What Does the Evidence Show? OJIN: The Online Journal of Issues in Nursing, 20, 2.

[7] (September 2017) State Laws: Training for Health Professionals in Suicide Assessment, Treatment, and Management. AFSP Public Policy Office, Washington DC. http://afsp.org/wp-content/uploads/2016/04/Health-Professional-Training-Issue-Br ief-9-7-17.pdf

[8] National Institute of Mental Health (2019) Suicide. https://www.nimh.nih.gov/health/statistics/suicide.shtml

[9] Stone, D.M., Simon, T.R., Fowler, K.A., Kegler, S.R., Yuan, K., Holland, K.M. and Crosby, A.E. (2018) Vital Signs: Trends in State Suicide Rates-United States, 1999-2016 and Circumstances Contributing to Suicide-27 States, 2015. Morbidity and Mortality Weekly Report, 67, 617-624.

https://www.cdc.gov/mmwr/volumes/67/wr/mm6722a1.htm\#suggestedcitation https://doi.org/10.15585/mmwr.mm6722a1

[10] Veisani, Y., Mohamadian, F. and Delpisheh, A. (2017) Prevalence and Comorbidity of Common Mental Disorders and Associations with Suicidal Ideation in the Adult Population. Epidemiology and Health, 39, e2017031.

https://doi.org/10.4178/epih.e2017031

[11] Lyons, M. (December 2018) Joint Commission Announces New National Patient Safety Goal to prevent suicide and Improve At-Risk Patient Care.

https://www.jointcommission.org/en/resources/news-and-multimedia/news/2018/1 2/joint-commission-announces-new-national-patient-safety-goal-to-prevent-suicid e-and-improve-atrisk-pa/

[12] Nicks, B.A. and Manthey, D.M. (2012) The Impact of Psychiatric Patient Boarding in Emergency Departments. Emergency Medicine International, 2012, Article ID: 360308. https://www.hindawi.com/journals/emi/2012/360308/ https://doi.org/10.1155/2012/360308

[13] Nordstrom, K., Berlin, J.S., Nash, S.S., Shah, S.B., Schmelzer, N.A. and Worley, L. 
(2019) Boarding of Mentally Ill Patients in Emergency Departments: American Psychiatric Association Resource Document. The Western Journal of Emergency Medicine, 20, 690-695. https://doi.org/10.5811/westjem.2019.6.42422

[14] Abid, Z., Meltzer, A., Lazar, D. and Pines, J. (2014) Psychiatric Boarding in U.S. EDs: A Multifactorial Problem That Requires Multidisciplinary Solutions. Policy Brief, 1, 1-6.

https://smhs.gwu.edu/urgentmatters/sites/urgentmatters/files/Psychiatric\%20Board ing\%20in\%20U.S.\%20EDs\%20A\%20Multifactorial\%20Problem\%20that\%20Require s\%20Multidisciplinary\%20Solutions.pdf

[15] Tavernero, T. (n.d.) Addressing Psychiatric Boarding in the Emergency Department. TEAMHealth.

http://www.teamhealth.com/wp-content/uploads/2018/09/white-paper-em-psychiat ric-boarding-in-the-ed.pdf

[16] ACEP Emergency Medicine Practice Committee, Ed. (2015) Practical Solutions to Boarding of Psychiatric Patients in the Emergency Department.

https://www.macep.org/Files/Behavioral\%20Health\%20Boarding/Practical\%20Solut ions\%20to\%20Boarding\%20of\%20Psych\%20Patients\%20in\%20EDs.pdf

[17] Schears, R. and Schears, M. (2019) Suicide Assessment and Disposition. https://www.reliasmedia.com/articles/143792-suicide-assessment-and-disposition

[18] Wilson, M., Nordstrom, K. and Zeller, S. (2013) Practical Management of the Suicidal Patient in the Emergency Department.

https://www.reliasmedia.com/articles/31408-practical-management-of-the-suicidalpatient-in-the-emergency-department

[19] National Institute of Mental Health (2017) State of Suicide Prevention in Emergency Care.

https://www.nimh.nih.gov/news/events/2017/state-of-suicide-prevention-in-emerge ncy-care.shtml

[20] McKenzie, H. (January 2018) Nurse Practitioners with Independent Practice Can Help Improve Emergency Department Quality Measures. https://pdfs.semanticscholar.org/8b65/de8fbfb6b9589931ae5f25a7a28eebfd0840.pdf

[21] Arias, S.A., Boudreaux, E.D., Segal, D.L., Miller, I., Camargo, C.A. and Betz, M.E. (2017) Disparities in Treatment of Older Adults with Suicide Risk in the Emergency Department. Journal of the American Geriatrics Society, 65, 2272-2277. https://onlinelibrary.wiley.com/doi/full/10.1111/igs.15011 https://doi.org/10.1111/jgs.15011

[22] Ramchand, R. and Becker, A. (2014) Suicide Rates in California: Trends and Implications for Prevention and Early Intervention Programs.

https://doi.org/10.7249/RB9737 